\title{
Spillover adherence effects of fixed-dose combination HIV therapy
}

\author{
This article was published in the following Dove Press journal: \\ Patient Preference and Adherence \\ 27 February 2012 \\ Number of times this article has been viewed
}

\author{
Teresa L Kauf' \\ Keith L Davis ${ }^{2}$ \\ Stephanie R Earnshaw ${ }^{2}$ \\ E Anne Davis ${ }^{3}$ \\ 'Department of Pharmaceutical \\ Outcomes and Policy, College \\ of Pharmacy, University of Florida, \\ Gainesville, FL, ${ }^{2} \mathrm{RTI}$ Health Solutions, \\ Research Triangle Park, NC, \\ ${ }^{3}$ Independent consultant, Pittsboro, \\ NC, USA
}

\begin{abstract}
The impact of fixed-dose combination (FDC) products on adherence to other, non-fixed regimen components has not been examined. We compared adherence to a third antiretroviral (ART) component among patients receiving a nucleoside reverse transcriptase inhibitor (NRTI) backbone consisting of the FDC Epzicom ${ }^{\circledR}$, GlaxoSmithKline Inc, Research Triangle Park, NC (abacavir sulfate $600 \mathrm{mg}+$ lamivudine $300 \mathrm{mg}$; FDC group) versus NRTI combinations taken as two separate pills (NRTI Combo group) using data from a national sample of 30 health plans covering approximately 38 million lives from 1997 to 2005. Adherence was measured as the medication possession ratio (MPR). Multivariate logistic regression compared treatment groups based on the likelihood of achieving $\geq 95 \%$ adherence, with sensitivity analyses using alternative thresholds. MPR was assessed as a continuous variable using multivariate linear regression. Covariates included age, gender, insurance payer type, year of study drug initiation, presence of mental health and substance abuse disorders, and third agent class. The study sample consisted of 650 FDC and 1947 NRTI Combo patients. Unadjusted mean adherence to the third agent was higher in the FDC group than the NRTI Combo group ( 0.92 vs $0.85 ; P<0.0001$ ). In regression analyses, FDC patients were $48 \%$ and $39 \%$ more likely to achieve $95 \%$ and $90 \%$ third agent adherence, respectively $(P \leq 0.03)$. None of the other MPR specifications achieved comparable results. Among managed care patients, use of an FDC appears to substantially improve adherence to a third regimen component and thus the likelihood of achieving the accepted standard for adherence to HIV therapy of $95 \%$.
\end{abstract}

Keywords: adherence, fixed-dose combination, antiretroviral

\section{Introduction}

Mortality and morbidity associated with HIV disease are dramatically reduced through the use of combination antiretroviral therapy (ART), commonly comprised of two nucleoside reverse transcriptase inhibitors (NRTIs) and either a non-NRTI (NNRTI) or a protease inhibitor (PI). ${ }^{1,2}$ The latter is often "boosted" with a low-dose of the PI, ritonavir (Norvir, Abbott Laboratories, Abbott Park, IL). ART regimens containing a boosted PI may consist of up to four separate pills, with several dosing frequencies and requirements. However, more compact ART regimens can be created by taking advantage of fixed-dose combination (FDC) products which consist of two or more antiretroviral drugs in a single tablet.

Since the introduction of the first FDC antiretroviral product, FDCs have become a mainstay in the treatment of HIV. The chief advantage of FDCs lies in simplifying a patient's regimen. In addition to reducing pill burden, many FDCs also have reduced dosing frequency and/or dosing requirements compared to other drugs in the FDC's
Correspondence: Teresa L Kauf PO Box 100496, Gainesville, FL 326I0 0496, USA

Tel +l 3522736252

Fax + I 3522736270

Email tkauf@ufl.edu which permits unrestricted noncommercial use, provided the original work is properly cited. 
therapeutic class. These factors work together to increase the likelihood of adherence. ${ }^{3}$ To date, most FDC introductions for HIV treatment have consisted of combinations of NRTIs which form the backbone of the ART regimen. ${ }^{4}$ Patients receiving an NRTI FDC would require a separate NNRTI or PI component.

In HIV, perhaps more than other chronic diseases, regimen adherence is a critical and necessary component of successful treatment. Previous studies have documented that adherence rates in excess of $95 \%$ are required in order to achieve a high likelihood of reaching an undetectable plasma HIV RNA level (viral load), a primary objective of ART. ${ }^{4-6}$ This high level of adherence is needed throughout the course of therapy to maintain undetectable viral load and to protect against the development of viral resistance to regimen components. Poor adherence and the subsequent development of resistance is a primary cause of regimen failure. ${ }^{4}$

FDCs have been associated with improved adherence compared to their separate components in both clinical trial and observational studies. For example, adherence to the FDC containing lamivudine $150 \mathrm{mg}+$ zidovudine $300 \mathrm{mg}$ (Combivir $^{\circledR}$, GlaxoSmithKline Inc, Research Triangle Park, NC) has been shown to be higher than adherence to its individual components. ${ }^{7,8}$ Similar results have been documented for FDCs in other therapeutic areas. ${ }^{9}$ In addition, it has been shown that a reduction in the total number of pills prescribed can improve adherence to the overall HIV regimen ${ }^{10-12}$ and suggested that improved adherence to an ART backbone also may result in improved regimen adherence. ${ }^{13-15}$ Yet, specific evidence of a spillover adherence effect on the remaining regimen components among individuals receiving FDCbased ART regimens is lacking. We compared adherence to the remaining regimen component among individuals receiving ART including an NRTI backbone containing an FDC of abacavir sulfate (ABC) $600 \mathrm{mg}+$ lamivudine (3TC) 300 mg (Epzicom, GlaxoSmithKline Inc, Research Triangle Park, NC) versus NRTI combinations given as two separate pills.

\section{Materials and methods Data and patients}

Data were taken from the Integrated Health Care Information Services (IHCIS, Waltham, MA) Managed Care Benchmark Database, which contains medical (inpatient, outpatient, physician, other ancillary) and pharmacy claims from a national sample of 30 managed care health plans covering approximately 38 million lives in the United States over the period 1997 to $2005 .{ }^{16}$ The IHCIS database has a representative distribution of age and gender compared with national enrollment in managed care plans. Geographic representation, however, is biased toward the East Coast. Patient-level records in the IHCIS database include demographics and information on benefits eligibility, and periods of health plan enrollment. Claims records in the IHCIS database contain detailed information on diagnoses, hospitalizations, diagnostic testing, therapeutic procedures, inpatient and outpatient physician services, prescription drug use, and cost data in the form of standardized managed care reimbursed amounts for each service. All claims in the IHCIS database were linked using unique patient identifiers and arranged in chronological order, thereby providing a detailed longitudinal file of all medical and pharmacy services utilized by each patient.

All patients in the IHCIS database with at least one pharmacy claim for Epzicom or for two or more components of an NRTI backbone as separate pills were eligible for study inclusion. For each patient, the date of the first prescription claim for any of these agents was designated the index date. Patients initiating Epzicom were designated the FDC group, while subjects receiving separate NRTI components on the index date were designated the NRTI Combo group. Patients in the NRTI Combo group were subject to the additional requirement that the first prescription for each drug be received within 7 days of the other in order to capture the intent to treat with a combination of separate pills. Patients in the FDC group were required to have at least one Epzicom refill within 60 days following the index date. Likewise, patients in the NRTI Combo group were required to have at least one refill for each agent within 60 days following initial receipt of the agent. Individuals in both treatment groups were excluded from the analysis if they met any of the following conditions: receipt of both Epzicom and one or more of its components on the index date; receipt of alternative therapy (ie, NRTI combo for the FDC group, or Epzicom for the NRTI Combo group) prior to the index date; failure to receive a third regimen component within 7 days of the index date; less than 6 months of continuous health plan enrollment prior to the index date; less than 60 days of continuous health plan enrollment after the index date; and/or age less than 18 years on the index date. The analysis was not limited to ART-naïve individuals because such patients may not accurately reflect the clinical populations using these drugs. However, because experienced and naïve patients may differ in their adherence behaviors, we control for prior ART exposure in the statistical analysis.

For each patient, a follow-up period was defined as the period beginning with the index date and ending with 
expiration of the days' supply for the last observed refill for the study therapy of interest, receipt of alternative study therapy (eg, Epzicom for the NRTI Combo group or vice versa), a gap of more than 180 days in study therapy, end of health plan enrollment, or end of the IHCIS database, whichever occurred first. Adherence to the remaining regimen component(s), as well as to the overall ART regimen, was assessed for each patient within the follow-up period. The remaining regimen components were categorized by class as PI, NRTI (excluding the NRTI backbone agents), NNRTI, entry inhibitor (EI), or a boosted PI. For simplicity, we refer to the remaining regimen component as the third agent. For patients who received more than three agents, one class among the non-NRTI backbone antiretrovirals in the regimen was randomly selected as the third agent for analysis purposes.

\section{Adherence measure}

The primary outcome evaluated in this study was treatment adherence to the non-NRTI-backbone regimen component as measured by the medication possession ratio (MPR). A recent systematic literature review found MPR to be the most widely adopted measure in published claims-based analyses of medication adherence (57\% of all studies). ${ }^{17}$ MPR is generally defined as the proportion of days within an observation period covered by the total days' supply obtained for a particular study drug within the observation period:

$$
\text { MPR }=\frac{\text { Sum of days' supply in observation period }}{\text { Days in observation period }}
$$

As noted in Andrade et al, ${ }^{17}$ a common observation period used in the MPR calculation is the number of days between the first dispense date and end of the days' supply of the last refill for the study therapy of interest. The same observation period was employed here. ${ }^{17}$

Total days supplied for the third agent was calculated as the combined days' supplied for all PIs, NNRTIs, EIs, and third NRTIs utilized during the follow-up period. We allowed patients to switch drugs within the same class for the third agent as the class in which that component was initiated (eg, PI to PI, NNRTI to NNRTI, etc) and control for this in the multivariate regressions. Table 1 presents the percentage of patients in each treatment group who switched within the third agent class (37\% of the FDC group, $27 \%$ of the NRTI combo group). Follow-up for third component adherence evaluation ended upon third agent switch to a drug outside of the class initiated (eg, upon switch from PI to NNRTI,
Table I Patient characteristics at baseline by treatment group

\begin{tabular}{|c|c|c|c|c|}
\hline & \multicolumn{4}{|c|}{ Treatment group } \\
\hline & \multicolumn{2}{|c|}{ FDC } & \multicolumn{2}{|c|}{ NRTI Combo } \\
\hline & $\mathbf{N}$ & (\%) & $\mathbf{N}$ & (\%) \\
\hline Total study sample & 650 & 100.0 & 1947 & 100.0 \\
\hline \multicolumn{5}{|l|}{ Gender } \\
\hline Male & 550 & 84.6 & 1597 & 82.0 \\
\hline Female & 100 & 15.4 & 350 & 18.0 \\
\hline \multicolumn{5}{|l|}{ Age category } \\
\hline $18-34$ & 96 & 14.8 & 329 & 16.9 \\
\hline $35-44$ & 278 & 42.8 & 920 & 47.3 \\
\hline $45-54$ & 205 & 31.5 & 534 & 27.4 \\
\hline $55-64$ & 62 & 9.5 & 142 & 7.3 \\
\hline$\geq 65$ & 9 & 1.4 & 22 & I.I \\
\hline \multicolumn{5}{|l|}{ Geographic region* } \\
\hline Northeast & 263 & 40.5 & 1106 & 56.8 \\
\hline South & 237 & 36.5 & 404 & 20.8 \\
\hline Midwest & 62 & 9.5 & 114 & 5.9 \\
\hline West & 69 & 10.6 & 82 & 4.2 \\
\hline Unknown & 19 & 2.9 & 241 & 12.4 \\
\hline \multicolumn{5}{|l|}{ Insurance payer type } \\
\hline Commercial & 635 & 97.7 & 1895 & 97.3 \\
\hline Medicaid & 8 & 1.2 & 28 & 1.4 \\
\hline Medicare & 7 & 1.1 & 24 & 1.2 \\
\hline \multicolumn{5}{|l|}{ Insurance product type* } \\
\hline $\begin{array}{l}\text { Health maintenance } \\
\text { organization }\end{array}$ & 231 & 35.5 & 699 & 35.9 \\
\hline Point of service plan & 145 & 22.3 & 249 & 12.8 \\
\hline $\begin{array}{l}\text { Preferred provider } \\
\text { organization }\end{array}$ & 266 & 40.9 & 915 & 47.0 \\
\hline Other & 8 & 1.2 & 84 & 4.3 \\
\hline \multicolumn{5}{|l|}{ Year of study drug initiation* } \\
\hline 1997 & - & - & 14 & 0.7 \\
\hline 1998 & - & - & 13 & 0.7 \\
\hline 1999 & - & - & 137 & 7.0 \\
\hline 2000 & - & - & 185 & 9.5 \\
\hline 2001 & - & - & 162 & 8.3 \\
\hline 2002 & - & - & 248 & 12.7 \\
\hline 2003 & - & - & 296 & 15.2 \\
\hline 2004 & 119 & 18.31 & 547 & 28.1 \\
\hline 2005 & 357 & 54.92 & 245 & 12.6 \\
\hline 2006 & 174 & 26.77 & 100 & 5.1 \\
\hline Prior ART in 6-month & 370 & 56.92 & 570 & 29.28 \\
\hline \multicolumn{5}{|l|}{ pre-index period* } \\
\hline \multicolumn{5}{|l|}{ MPR regimen } \\
\hline Mean (SD)** & 0.88 & 0.170 & 0.82 & 0.20 \\
\hline Median (range) & 0.96 & $0.26-1.00$ & 0.86 & $0.08-1.00$ \\
\hline$M P R \geq 0.80 * *$ & 502 & 77.2 & 1269 & 65.2 \\
\hline$M P R \geq 0.85^{* *}$ & 459 & 70.6 & 1126 & 57.8 \\
\hline$M P R \geq 0.90^{* *}$ & 426 & 65.5 & 953 & 49.0 \\
\hline$M P R \geq 0.95^{* *}$ & 355 & 54.6 & 766 & 39.3 \\
\hline \multicolumn{5}{|l|}{ MPR third component } \\
\hline Mean $(S D)^{* *}$ & 0.92 & 0.168 & 0.85 & 0.245 \\
\hline Median (range) & 1.0 & $0.03-1.0$ & 0.98 & $0.005-1.0$ \\
\hline$M P R \geq 0.80 * *$ & 565 & 86.9 & 1460 & 75.0 \\
\hline$M P R \geq 0.85^{* *}$ & 542 & 83.4 & 1362 & 70.0 \\
\hline$M P R \geq 0.90^{* *}$ & 514 & 79.1 & 1250 & 64.2 \\
\hline $\mathrm{MPR} \geq 0.95^{* *}$ & 478 & 73.5 & 1103 & 56.7 \\
\hline Switched within third agent class & 242 & 37.2 & 522 & 26.8 \\
\hline
\end{tabular}

Note: *Indicates differences between groups at $P<0.001$; $* * P<0.000$ I.

Abbreviations: ART, antiretroviral therapy; FDC, fixed-dose combination; MPR, medication possession ratio; NRTI, nucleoside reverse transcriptase inhibitor; SD, standard deviation. 
NNRTI to PI). Days covered by both the NRTI backbone and the third agent were assessed by evaluating overlapping days' supply for the respective agents.

\section{Statistical analysis}

Patient characteristics were measured at the index date and included age, gender, geographic region, insurance payer type (eg, commercial, self, or government-sponsored), insurance product type (eg, health maintenance organization [HMO], preferred provider organization [PPO]), prior exposure to antiretroviral therapy, and eligibility for mental health benefits coverage. We also assessed the presence of substance abuse and mental health disorders that have been linked to poor ART adherence during the 6-month period prior to therapy initiation. ICD-9 diagnostic and CPT-4 procedural codes appearing in medical records were used to identify these conditions and were based on algorithms set forth by the US Substance Abuse and Mental Health Services Administration (SAMHSA). ${ }^{18}$

Descriptive analyses of patient characteristics and unadjusted adherence measures were conducted as mean values and standard deviations for continuous variables of interest and frequency distributions for categorical variables of interest. The statistical significance of descriptive differences in adherence outcomes between the FDC and NRTI Combo groups, as well as between patients who are adherent and not adherent with each study therapy based on relevant MPR thresholds, were measured using t-tests and $\chi^{2}$ tests as appropriate, with results reported as $P$ values.

To assess differences in adherence to the third agent between the FDC and NRTI Combo groups, multivariate regressions of the following general forms were estimated:

$$
\begin{gathered}
\text { MPR }=\beta_{0}+\beta_{1} \text { FDC }+\beta_{2} X_{i}+\varepsilon, \text { and } \\
\text { ADHERENT }=\beta_{0}+\beta_{1} \text { FDC }+\beta_{2} X_{i}+\varepsilon .
\end{gathered}
$$

In Equation 1, continuous MPR was estimated in a linear model as a function of a dichotomous indicator FDC equal to one for patients who initiated Epzicom and zero for patients who initiated an NRTI backbone as separate components, and a vector of baseline patient characteristics $\left(\mathrm{X}_{\mathrm{i}}\right)$. Equation 2 was a logistic model with the same covariate structure as Equation 1. The dichotomous outcome, ADHERENT, was set equal to one for patients meeting one of four MPR thresholds (MPR $\geq 0.80$, $0.85,0.90$, and 0.95 ) and zero for patients not meeting the threshold. This range of values was chosen based on previous evidence suggesting that lower risk of virologic failure is associated with maintaining adherence of at least 95\%., 5,6
All analyses were carried out using $\mathrm{SAS}^{\circledR}$ (v 9; SAS Institute, Cary, NC) statistical software.

\section{Results}

After applying inclusion and exclusion criteria as described previously, there were 650 individuals in the FDC group and 1947 in the NRTI Combo group (Figure 1). In both groups, the proportions of patients receiving an NNRTI or a PI as the third agent of their regimens were similar (Table 2). Roughly $59 \%, 42 \%$, and $37 \%$ of patients in the NRTI group received lamivudine, tenofovir, or stavudine, respectively. Median duration of follow-up for the FDC group was 272 days; for the NRTI Combo group, 338 days.

The distribution of baseline (index date) characteristics for patients in the FDC and NRTI Combo groups is shown in Table 1 . The majority of study subjects were aged $35-54$ years, and $82.7 \%$ were men. FDC group patients had an unadjusted mean adherence of 0.88 (standard deviation $[\mathrm{SD}]=0.17)$ versus $0.82(\mathrm{SD}=0.20)$ for the NRTI Combo, with median adherence of 0.96 (range: $0.26-1.00$ ) versus 0.89 (range: $0.08-1.00)$, respectively $(P<0.0001$ for mean comparisons). Mean adherence to the third agent was 0.923 $(\mathrm{SD}=0.17)$ for the FDC group and $0.85(\mathrm{SD}=0.25)$ for the NRTI Combo group $(P<0.0001)$.

Partial results for the multivariate regression models of third agent adherence, as described below, are shown in Table 3; full results are available upon request from the authors. In multivariate logistic analyses, the presence of an FDC NRTI backbone consistently improved the likelihood of adherence at the four thresholds examined, and this impact was statistically significant at the $90 \%$ and $95 \%$ adherence thresholds. Patients in the FDC group were 47\% more likely to achieve a clinically meaningful adherence level of $95 \%$ to the third agent compared to the NRTI Combo group. When the threshold for adherence was considered at the lower $90 \%$ level, the likelihood of achieving that level of adherence to the third agent was $40 \%$ higher among FDC patients. Similarly, when adherence was defined as a continuous variable, use of an FDC provided a small improvement in adherence to the third agent compared to the use of separate pills (NRTI Combo), but the magnitude of the effect did not reach statistical significance.

Relative to the PI class, third agents that were from the NRTI or boosted PI classes were associated with a higher likelihood of adherence (or better odds of achieving a certain level of adherence) in all three models shown in Table 3. In the continuous MPR model, NRTIs were associated with an absolute increase in adherence of $11.6 \%$; boosted PIs, $7.3 \%$. 


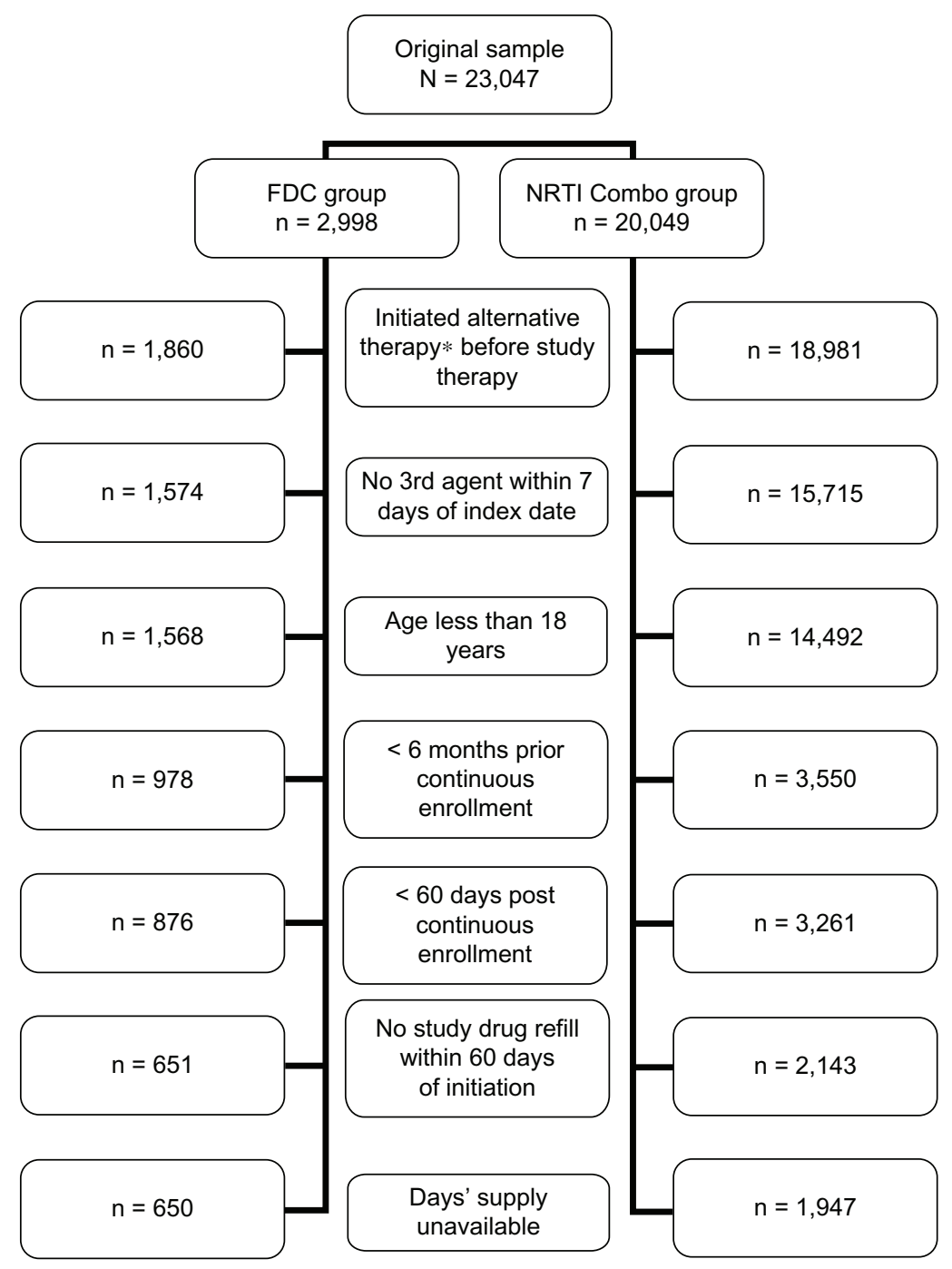

Figure I Sequential application of study inclusion and exclusion criteria to the IHCIS sample.

Note: *Alternative therapy use refers to the use of a non-study drug within the group (eg, the use of Epzicom by the NRTI Combo group).

Abbreviations: FDC, fixed-dose combination; IHCIS, Integrated Health Care Information Services; NRTI, nucleoside reverse transcriptase inhibitor.

Entry inhibitors were consistently associated with reduced adherence compared to PIs, but the effect reached only near statistical significance in the continuous MPR model. Switching of the third agent within the same class was also consistently associated with improved adherence in all three models. Prior ART experience in the 6-month, pre-index period was insignificant across all models.

\section{Discussion}

Evidence from clinical trials and observational studies in a variety of therapeutic applications supports the ability of FDC products to improve adherence to therapy, compared to separate pills. ${ }^{7,919,20}$ To our knowledge, this is the first study to empirically quantify the spillover effect of FDCs in improving adherence to another regimen component.
Our results confirm the hypothesis that use of an FDC as the backbone of an ART regimen improves adherence to the third agent, compared to a regimen that utilizes separate pills as a regimen backbone. This improvement in adherence is in addition to the increase associated solely with the backbone component of the regimen.

While some evidence suggests that newer antiretrovirals and ART combinations may be more "forgiving" of poor adherence than older agents and un-boosted regimens, the standard for adherence in HIV remains high. ${ }^{4}$ In our population of managed care enrollees, patients receiving an FDC backbone were nearly 50\% more likely to achieve an adherence rate of $95 \%$ or better to the third agent in the regimen. Even at the lower $90 \%$ threshold, FDC backbone use was associated with a nearly $40 \%$ increase in the likelihood of 
Table 2 Antiretroviral therapy use by treatment group

\begin{tabular}{|c|c|c|c|c|}
\hline & \multicolumn{4}{|c|}{ Treatment group } \\
\hline & \multicolumn{2}{|c|}{ FDC } & \multicolumn{2}{|c|}{ NRTI Combo } \\
\hline & $\mathbf{N}$ & (\%) & $\mathbf{N}$ & (\%) \\
\hline \multicolumn{5}{|l|}{ NRTI Combo initiated } \\
\hline Lamivudine + stavudine & - & - & 500 & 25.7 \\
\hline Lamivudine + tenofovir & - & - & 377 & 19.4 \\
\hline Tenofovir + emtricitibine & - & - & 188 & 9.7 \\
\hline Tenofovir + didanosine & - & - & 125 & 6.4 \\
\hline Lamivudine + abacavir & - & - & 113 & 5.8 \\
\hline Stavudine + didanosine & - & - & 104 & 5.3 \\
\hline Lamivudine + didanosine & - & - & 91 & 4.7 \\
\hline Lamivudine + zidovudine & - & - & 70 & 3.6 \\
\hline Tenofovir + abacavir & - & - & 67 & 3.4 \\
\hline Stavudine + abacavir & - & - & 62 & 3.2 \\
\hline Tenofovir + stavudine & - & - & 53 & 2.7 \\
\hline Other combinations & - & - & 197 & 10.1 \\
\hline \multicolumn{5}{|c|}{ Thirrd ART component initiated (drug class) } \\
\hline $\mathrm{PI}$ (alone) & 194 & 29.9 & 637 & 32.7 \\
\hline NNRTI & 53 & 8.2 & 72 & 3.7 \\
\hline NRTI* & 262 & 40.3 & 987 & 50.7 \\
\hline PI (w/ritonavir booster) & 4 & 0.62 & 3 & 0.15 \\
\hline $\mathrm{El}$ & 137 & 21.1 & 248 & 12.7 \\
\hline \multicolumn{5}{|c|}{ Third ART component initiated (drug name) } \\
\hline Efavirenz & 203 & 31.2 & 757 & 38.9 \\
\hline Atazanavir & 161 & 24.8 & 215 & 11.0 \\
\hline Lopinavir/ritonavir FDC & 67 & 10.3 & 279 & 14.3 \\
\hline Lexiva (w/Norvir booster) & 51 & 7.9 & 28 & 1.4 \\
\hline Nevirapine & 59 & 9.1 & 224 & 11.5 \\
\hline Tenofovir & 31 & 4.8 & 29 & 1.5 \\
\hline Fosamprenavir & 18 & 2.8 & 20 & 1.0 \\
\hline Ritonavir (full-dose) & 11 & 1.7 & 21 & I.I \\
\hline Stavudine & 8 & 1.2 & 1 & 0.1 \\
\hline Didanosine & 6 & 0.9 & I & 0.1 \\
\hline Nelfinavir & 7 & I.I & 157 & 8.1 \\
\hline Indinavir & 4 & 0.6 & 93 & 4.8 \\
\hline Lamivudine & 1 & 0.2 & 7 & 0.4 \\
\hline Other & 23 & 3.5 & 115 & 5.9 \\
\hline
\end{tabular}

Note: *For the NRTI Combo group, the third NRTI initiated must be different from either of the two NRTIs initiated.

Abbreviations: NRTI, nucleoside reverse transcriptase inhibitor; Pl, protease inhibitor; NNRTI, non-nucleoside reverse transcriptase inhibitor; El, entry inhibitor; $A R T$, antiretroviral therapy; FDC, fixed-dose combination.

third-agent adherence. The magnitude of the FDC spillover adherence effect observed in our sample, while smaller than the approximately $300 \%$ direct effect estimated by Legorreta et al, ${ }^{8}$ is nonetheless statistically and clinically meaningful.

Results for adherence thresholds of $80 \%$ and $85 \%$ and for continuous adherence were also positive, but did not reach statistical significance. The inability to detect a spillover effect in these other model specifications may reflect the adherence behavior of our sample. Mean regimen adherence among the patients in this study was $88 \%$, relatively high compared to other reports, though not unprecedented. ${ }^{21}$ Examination of adherence effects from FDCs in patient populations with lower baseline levels of adherence is needed to fully understand the spillover phenomenon.

Across the spectrum of available antiretrovirals, adherence is positively correlated with virologic suppression. ${ }^{4}$ FDC backbones, then, may play an important role in improving adherence and likelihood of suppression for the remaining regimen component(s), regardless of drug class. Additionally, adherence plays an important role in the development of drug resistance, although the specific relationships may differ across drug classes. ${ }^{22}$ As such, the spillover adherence effect of FDCs may be especially clinically meaningful for PI-based regimens. Studies examining the relationship between adherence and resistance suggest that the development of primary and secondary PI mutations peaks at levels of adherence just below those required to maintain virologic suppression..$^{22,23}$ For example, among patients with relatively high overall adherence, even small, absolute improvements in PI adherence of only a few percentage points may be sufficient to substantially reduce mutation development. The use of an FDC backbone may be one tool for achieving such an improvement in PI adherence.

The use of administrative records to examine the spillover effect of FDCs offers several advantages over other data sources. First, these data reflect the experiences of patients in actual clinical practice, potentially avoiding the adherence bias associated with clinical trials. ${ }^{24}$ Second, these observational data allow for a relatively large sample size, thereby increasing the ability to detect differences between study groups. Additionally, while not a nationally representative sample, the IHCIS data include individuals from all regions of the US. To the extent that heterogeneity in adherence is correlated with regional heterogeneity in HIV risk, demographic characteristics, and other factors that may affect adherence, the generalizability of our results is improved relative to smaller studies of more homogeneous cohorts.

There are differences between the IHCIS sample, and the US HIV population as a whole. In 2006, approximately $73 \%$ of individuals living with a diagnosis of HIV or AIDS in the US in 2006 were males, compared to nearly $83 \%$ in our study ${ }^{25}$ During the same time period, roughly $38 \%$ of persons living with HIV/AIDS were aged 18-34 years, compared to less than $15 \%$ in our sample. The older age and increased proportion of males observed in our sample may reflect an increased access to health insurance for this group compared to other individuals diagnosed with HIV/AIDS. It is possible that spillover effects measured in different populations may differ from those seen in the IHCIS sample, especially if factors associated with insurance status, such as education and 
Table 3 Results of multivariate regression analyses on third component adherence

\begin{tabular}{|c|c|c|c|c|c|c|}
\hline \multirow[t]{2}{*}{ Covariates } & \multicolumn{2}{|c|}{ Continuous MPR } & \multicolumn{2}{|l|}{ MPR $\geq 90 \%$} & \multicolumn{2}{|l|}{ MPR $\geq 95 \%$} \\
\hline & $\begin{array}{l}\text { Parameter } \\
\text { estimate (SE) }\end{array}$ & $P$ value & Odds ratio & $95 \% \mathrm{Cl}$ & Odds ratio & $95 \% \mathrm{Cl}$ \\
\hline FDC group (vs NRTI Combo) & $0.0155(0.0132)$ & 0.2406 & 1.40 & $1.05-1.86$ & 1.47 & $1.12-1.94$ \\
\hline Male (vs Female) & $0.0133(0.0116)$ & 0.2545 & 1.21 & $0.95-1.53$ & 1.25 & $0.99-1.57$ \\
\hline \multicolumn{7}{|l|}{ Age (vs I8-34) } \\
\hline $35-44$ & $0.0047(0.0125)$ & 0.7042 & 0.90 & $0.70-1.16$ & 0.86 & $0.67-I . I I$ \\
\hline $45-54$ & $0.0173(0.0134)$ & 0.2010 & 1.04 & $0.79-1.37$ & 0.98 & $0.75-1.29$ \\
\hline $55-64$ & $0.0257(0.0189)$ & 0.1729 & 0.95 & $0.64-1.40$ & 1.13 & $0.77-1.66$ \\
\hline$\geq 65$ & $0.0822(0.0420)$ & 0.0507 & $\mathrm{I} .47$ & $0.60-3.58$ & 1.67 & $0.70-3.96$ \\
\hline \multicolumn{7}{|l|}{ Location (vs Northeast) } \\
\hline South & $-0.0236(0.0127)$ & 0.0631 & 0.68 & $0.53-0.89$ & 0.65 & $0.50-0.84$ \\
\hline Midwest & $0.0258(0.0189)$ & 0.1739 & 1.37 & $0.91-2.01$ & 1.00 & $0.68-1.46$ \\
\hline West & $0.0404(0.0205)$ & 0.0489 & 1.48 & $0.94-2.32$ & 1.24 & $0.81-1.89$ \\
\hline Unknown & $0.0043(0.0153)$ & 0.7784 & 1.07 & $0.78-1.45$ & 0.90 & $0.67-1.22$ \\
\hline Commercial payer (vs public) & $0.0733(0.0293)$ & 0.0125 & 2.93 & $|.6|-5.3 \mid$ & 2.19 & $1.20-3.98$ \\
\hline \multicolumn{7}{|l|}{ Plan type (vs POS) } \\
\hline $\mathrm{HMO}$ & $0.0065(0.0141)$ & 0.6445 & 1.17 & $0.87-1.57$ & 1.18 & $0.89-1.58$ \\
\hline PPO & $-0.0091(0.0136)$ & 0.5052 & 0.95 & $0.72-1.27$ & 0.98 & $0.74-1.30$ \\
\hline Other & $-0.0180(0.0266)$ & 0.4998 & 1.12 & $0.65-1.94$ & 1.27 & $0.74-2.17$ \\
\hline \multicolumn{7}{|l|}{ Year of ART initiation (vs 1997) } \\
\hline 1998 & $-0.0698(0.0843)$ & 0.4077 & 0.74 & $0.15-3.55$ & 0.52 & $0.10-2.65$ \\
\hline 1999 & $-0.1324(0.0630)$ & 0.0355 & 0.40 & $0.12-1.33$ & 0.56 & $0.17-1.81$ \\
\hline 2000 & $-0.1309(0.0624)$ & 0.0361 & 0.47 & $0.14-1.56$ & 0.56 & $0.17-1.81$ \\
\hline 2001 & $-0.1625(0.0626)$ & 0.0095 & 0.34 & $0.10-1.14$ & 0.50 & $0.15-1.62$ \\
\hline 2002 & $-0.1405(0.0617)$ & 0.0229 & 0.38 & $0.12-1.24$ & 0.54 & $0.17-1.73$ \\
\hline 2003 & $-0.0854(0.0616)$ & 0.1657 & 0.57 & $0.17-1.87$ & 0.82 & 0.7360 \\
\hline 2004 & $-0.0419(0.0602)$ & 0.4859 & 0.87 & $0.27-2.77$ & 1.18 & $0.38-3.67$ \\
\hline 2005 & $-0.0356(0.0604)$ & 0.5553 & 0.80 & $0.25-2.57$ & 0.97 & $0.31-3.02$ \\
\hline 2006 & $-0.0026(0.0614)$ & 0.9664 & 1.09 & $0.33-3.60$ & 1.38 & $0.43-4.43$ \\
\hline Eligible for $\mathrm{MH}$ benefits & $0.0152(0.0140)$ & 0.2780 & 1.26 & $0.94-1.69$ & 1.14 & $0.86-1.52$ \\
\hline \multicolumn{7}{|l|}{ Prior diagnoses (vs None) } \\
\hline Prior $\mathrm{MH}$ & $0.0100(0.0124)$ & 0.4180 & 1.36 & $1.04-1.77$ & 1.55 & $1.20-2.00$ \\
\hline Prior SA & $-0.0095(0.0493)$ & 0.8477 & 1.23 & $0.4 I-3.68$ & 1.30 & $0.45-3.77$ \\
\hline Prior $\mathrm{MH}+\mathrm{SA}$ & $0.0177(0.0650)$ & 0.7856 & 0.79 & $0.19-3.32$ & 0.64 & $0.16-2.53$ \\
\hline \multicolumn{7}{|l|}{ Third agent class (vs PI) } \\
\hline NRTI & $0.1164(0.0242)$ & $<0.0001$ & 10.56 & $4.09-27.25$ & 9.74 & $4.06-23.35$ \\
\hline NNRTI & $0.0177(0.0103)$ & 0.0861 & 1.11 & $0.91-1.35$ & 1.12 & $0.93-1.36$ \\
\hline $\mathrm{El}$ & $-0.1623(0.0834)$ & 0.0518 & 0.74 & $0.16-3.52$ & 0.62 & $0.13-2.87$ \\
\hline Boosted PI & $0.0731(0.0182)$ & $<0.0001$ & 4.83 & $2.95-7.91$ & 5.45 & $3.36-8.84$ \\
\hline Switched within third agent class & $0.0476(0.0153)$ & 0.0019 & 1.72 & $1.27-2.33$ & 2.21 & $1.64-2.97$ \\
\hline Prior ART in 6-month pre-index period & $-0.0147(0.0095)$ & 0.1217 & 0.98 & $0.81-1.19$ & 1.01 & $0.84-1.23$ \\
\hline
\end{tabular}

Abbreviations: ART, antiretroviral therapy; $\mathrm{Cl}$, confidence interval; El, entry inhibitor; FDC, fixed-dose combination; $\mathrm{HMO}$, health maintenance organization; $\mathrm{MH}$, mental health; MPR, medication possession ratio; NRTI, nucleoside reverse transcriptase inhibitor; NNRTI, non-nucleoside reverse transcriptase inhibitor; PI, protease inhibitor; POS, point of service; PPO, preferred provider organization; SA, substance abuse; SE, standard error.

income (which are unobserved in the IHCIS sample), also influence adherence behavior. While socioeconomic status does appear to be associated with adherence in many chronic diseases, such relationships have not been documented conclusively for HIV. ${ }^{26}$

As with other observational studies, this work is subject to limitations which should be considered when evaluating the results. The retrospective nature of the data source raises the possibility of selection bias because the assignment of patients to study groups is not random, as in a clinical trial.
For example, if individuals who received Epzicom were more likely to be adherent to a third regimen component than those who received a backbone of separate NRTIs, the impact of FDCs on spillover adherence would be overestimated. Conversely, if physicians who were particularly concerned with patient adherence tended to prescribe FDCs to that end, then the spillover effect observed here may be underestimated. It should be noted that the study inclusion requirement of at least one refill in the 60 days following the index prescription - necessary to capture the intent to treat 
with a specific agent - may bias the sample toward more adherent patients. In such a case, the ability of the FDC to improve adherence even among those already adherent is notable. Still, further efforts are needed to understand adherence among very poor adherers.

As noted in Table 1, subjects in the two study groups were similar in terms of age and gender, but other differences did exist. We controlled for all the factors listed in Table 1 in our statistical analysis. We did not have access to subjects' complete HIV treatment histories and could not definitively identify those who were completely naïve to treatment. While there is some evidence that adherence differs between treatment-experienced and treatment-naïve patients, ${ }^{27}$ the likelihood that the marginal adherence effect estimated here would differ between these two groups of patients was less likely and, indeed, was what we observed. There may exist other factors for which we were unable to control. If those factors were associated with adherence differentially by study group, the study results may be biased.

Epzicom dosing is labeled as once per day, while most other NRTI combinations used during the study period were dosed twice per day, per product labeling. Thus, some of the spillover attributed to the FDC group may be due to reduced dosing frequency. However, some of the combinations in the NRTI Combo group included one or two agents that could be dosed once per day. Interestingly, patients in the NRTI Combo were more likely to receive an NNRTI as the third regimen component. Compared to PIs, NNRTIs have been associated with improved adherence. $^{28}$

The approach we use to construct the follow-up period over which adherence was assessed is subject to the inherent limitation that some patients may discontinue study therapy before expiration of the last observed refill. Since discontinuation in that case cannot be observed in automated pharmacy claims, our method may lead to follow-up durations that are slightly overestimated for some patients. Similarly, no gold standard for measurement of adherence exists. Adherence levels measured from pharmacy records have been shown to be higher than those measured by electronic monitoring, although the two measures are highly correlated..$^{29}$ Importantly, any overestimation of adherence would be attributable to both study groups, minimizing its effect.

This study examines only the use of an FDC containing two antiretrovirals and intended to be combined with at least one additional agent. Due to sample size constraints, we were unable to assess whether spillover effects extend to all components of regimens that consist of an FDC plus two or more antiretrovirals taken as separate pills. FDCs containing three antiretrovirals are also available and can be used alone or as part of a larger regimen in combination with other antiretrovirals. It is unclear, a priori, whether such effects would be greater or lesser than those seen with two-agent FDCs in a typical, three-agent regimen. We have shown that adherence to a third regimen component is increased with the use of an FDC backbone and that this adherence level is high. As a result, in populations where medication-taking behavior is relatively high, triple-agent FDCs may not offer much of an adherence advantage over dual-agent FDCs. Additional research on the direct and spillover adherence effects of dual-agent and triple-agent FDCs as components of regimens consisting of more than three antiretrovirals is warranted.

Successful HIV therapy requires individuals to maintain nearly perfect adherence, and clinical practice guidelines have long recommended that physicians consider adherence when selecting regimen components. This study highlights an additional adherence advantage associated with the use of FDCs that has not previously been identified: a spillover effect on a non-fixed regimen component. While additional efforts are needed to assess the extent of adherence spillover associated with other FDCs, our work supports the use of fixed-dose NRTI backbones as a means of encouraging adherence to the entire ART regimen.

\section{Acknowledgments}

The authors thank Christopher M Blanchette for his contributions to this study. This work was funded by GlaxoSmithKline Inc (Research Triangle Park, NC). The individual authors' contributions to the work are as follows: TLK led the study design, interpretation of results, and writing. As corresponding author, TLK takes full responsibility for the entirety of the content of the manuscript. KLD conducted all data analysis and contributed to the interpretation of results and writing. SRE assisted with study design and contributed to the interpretation of results. EAD provided the data source for the study and contributed to the study design.

\section{Disclosures}

This study was funded by a contract between GlaxoSmithKline Inc (Research Triangle Park, NC), which manufactures abacavir sulfate, zidovudine, lamivudine, and various combinations of these drugs, and RTI Health Solutions 
(Research Triangle Park, NC), an independent contract research organization that has received research funding for this and other studies from GlaxoSmithKline and other pharmaceutical companies that market drugs for use in HIV patients and other medical conditions. KLD and SRE are employees of RTI Health Solutions. At the time of the study, EAD was an employee of GlaxoSmithKline. TLK is an Associate Professor of Pharmaceutical Outcomes and Policy at the University of Florida. She has provided consultancy to RTI Health Solutions and has received funding for other studies from GlaxoSmithKline and other pharmaceutical companies that market drugs for use in the treatment of HIV patients.

\section{References}

1. Mocroft A, Ledergerber B, Katlama C; EuroSIDA study group, et al. Decline in the AIDS and death rates in the EuroSIDA study: an observational study. Lancet. 2003;362(9377):22-29.

2. Palella FJ Jr, Delaney KM, Moorman AC, et al. Declining morbidity and mortality among patients with advanced human immunodeficiency virus infection. HIV Outpatient Study Investigators. $N$ Engl $J$ Med. 1998;338(13):853-860.

3. Stone VE, Hogan JW, Schuman P; HERS study, et al. Antiretroviral regimen complexity, self-reported adherence, and HIV patients' understanding of their regimens: survey of women in the her study. $J$ Acquir Immune Defic Syndr. 2001;28(2):124-131.

4. Panel on Antiretroviral Guidelines for Adults and Adolescents. Guidelines for the use of antiretroviral agents in HIV-1-infected adults and adolescents. Department of Health and Human Services. October 14, 2011;1-167. Available from: http://www.aidsinfo.nih.gov/ContentFiles/AdultandAdolescentGL.pdf. Accessed on October 31, 2011.

5. Paterson DL, Swindells S, Mohr J, et al. Adherence to protease inhibitor therapy and outcomes in patients with HIV infection. Ann Intern Med. 2000;133(1):21-30.

6. Low-Beer S, Yip B, O’Shaughnessy MV, Hogg RS, Montaner JS. Adherence to triple therapy and viral load response. J Acquir Immune Defic Syndr. 2000;23(4):360-361.

7. Eron J Jr, Yeni P, Gathe J Jr; KLEAN study team, et al. The KLEAN study of fosamprenavir-ritonavir versus lopinavir-ritonavir, each in combination with abacavir-lamivudine, for initial treatment of HIV infection over 48 weeks: a randomised non-inferiority trial. Lancet. 2006;368(9534):476-482.

8. Legorreta A, Yu A, Chernicoff H, Gilmore A, Jordan J, Rosenzweig JC. Adherence to combined lamivudine + zidovudine versus individual components: a community-based retrospective medicaid claims analysis. AIDS Care. 2005;17(8):938-948.

9. Bangalore S, Kamalakkannan G, Parkar S, Messerli FH. Fixed-dose combinations improve medication compliance: a meta-analysis. Am J Med. 2007;120(8):713-719.

10. Gardner EM, Burman WJ, Maravi ME, Davidson AJ. Selective drug taking during combination antiretroviral therapy in an unselected clinic population. J Acquir Immune Defic Syndr. 2005;40(3): 294-300.

11. Maggiolo F, Ravasio L, Ripamonti D, et al. Similar adherence rates favor different virologic outcomes for patients treated with nonnucleoside analogues or protease inhibitors. Clin Infect Dis. 2005;40(1):158-163.

12. Portsmouth SD, Osorio J, McCormick K, Gazzard BG, Moyle GJ. Better maintained adherence on switching from twice-daily to oncedaily therapy for HIV: a 24-week randomized trial of treatment simplification using stavudine prolonged-release capsules. HIV Med. 2005;6(3):185-190.
13. Crémieux AC, Katlama C, Gillotin C; AZ110002 Study Group et al. A comparison of the steady-state pharmacokinetics and safety of abacavir, lamivudine, and zidovudine taken as a triple combination tablet and as abacavir plus a lamivudine-zidovudine double combination tablet by HIV-1-infected adults. Pharmacotherapy. 2001;21(4):424-430.

14. Lamarca A, Clumeck N, Plettenberg A, et al. Efficacy and safety of a once-daily fixed-dose combination of abacavir/lamivudine compared with abacavir twice daily and lamivudine once daily as separate entities in antiretroviral-experienced HIV-1-infected patients (CAL30001 Study). J Acquir Immune Defic Syndr. 2006;41(5):598-606.

15. Moore KH, Shaw S, Laurent AL, et al. Lamivudine/zidovudine as a combined formulation tablet: bioequivalence compared with lamivudine and zidovudine administered concurrently and the effect of food on absorption. J Clin Pharmacol. 1999;39(6):593-605.

16. Ingenix. Integrated Health Care Information Services. Available from: http://www.ingenix.com/AboutUs/Businesses/ihcis/.

17. Andrade SE, Kahler KH, Frech F, Chan KA. Methods for evaluation of medication adherence and persistence using automated databases. Pharmacoepidemiol Drug Saf. 2006;15(8):565-574.

18. Finkelstein E, Bray J, Larson M, et al. Mental health. In: Manderscheid RW, Henderson MJ, editors. Prevalence of, and payments for, mental health and substance abuse disorders in public and private sector health plans. Rockville, Maryland: United States Department of Health and Human Services, Center for Mental Health Services; 2002. Available from: http://store.samhsa.gov/product/Mental-Health-UnitedStates-2002/SMA04-3938. Accessed on June 19, 2009.

19. Melikian C, White TJ, Vanderplas A, Dezii CM, Chang E. Adherence to oral antidiabetic therapy in a managed care organization: a comparison of monotherapy, combination therapy, and fixed-dose combination therapy. Clin Ther. 2002;24(3):460-467.

20. Vanderpoel DR, Hussein MA, Watson-Heidari T, Perry A. Adherence to a fixed-dose combination of rosiglitazone maleate/metformin hydrochloride in subjects with type 2 diabetes mellitus: a retrospective database analysis. Clin Ther. 2004;26(12):2066-2075.

21. Maitland D, Jackson A, Osorio J, Mandalia S, Gazzard BG, Moyle GJ. Switching from twice-daily abacavir and lamivudine to the oncedaily fixed-dose combination tablet of abacavir and lamivudine improves patient adherence and satisfaction with therapy. HIV Med. 2008;9(8):667-672.

22. Bangsberg DR, Acosta EP, Gupta R, et al. Adherence-resistance relationships for protease and non-nucleoside reverse transcriptase inhibitors explained by virological fitness. AIDS. 2006;20(2):223-231.

23. King MS, Brun SC, Kempf DJ. Relationship between adherence and the development of resistance in antiretroviral-naive, HIV-1-infected patients receiving lopinavir/ritonavir or nelfinavir. $J$ Infect Dis. 2005;191(12):2046-2052.

24. White HD. Adherence and outcomes: it's more than taking the pills. Lancet. 2005;366(9502):1989-1991.

25. Centers for Disease Control and Prevention. HIV/AIDS Surveillance Report 2006. Available from: http://www.cdc.gov/hiv/surveillance/ resources/reports/2006report/. Accessed on January 23, 2009.

26. Falagas ME, Zarkadoulia EA, Pliatsika PA, Panos G. Socioeconomic status (SES) as a determinant of adherence to treatment in HIV infected patients: a systematic review of the literature. Retrovirology. 2008;5:13.

27. Horberg M, Silverberg M, Hurley L, Delorenze G, Quesenberry C. Influence of prior antiretroviral experience on adherence and responses to new highly active antiretroviral therapy regimens. AIDS Patient Care STDS. 2008;22(4):301-312.

28. Martin M, Del Cacho CE, Codina C, et al. Relationship between adherence level, type of the antiretroviral regimen, and plasma HIV type 1 RNA viral load: a prospective cohort study. AIDS Res Hum Retroviruses. 2008;24(10):1263-1268.

29. Hansen RA, Kim MM, Song L, Tu W, Wu J, Murray MD. Comparison of methods to assess medication adherence and classify nonadherence. Ann Pharmacother. 2009;43(3):413-422. 


\section{Publish your work in this journal}

Patient Preference and Adherence is an international, peer-reviewed, open access journal focusing on the growing importance of patient preference and adherence throughout the therapeutic continuum. Patient satisfaction, acceptability, quality of life, compliance, persistence and their role in developing new therapeutic modalities and compounds to

optimize clinical outcomes for existing disease states are major areas of interest. This journal has been accepted for indexing on PubMed Central. The manuscript management system is completely online and includes a very quick and fair peer-review system. Visit http://www.dovepress.com/ testimonials.php to read real quotes from published authors.

Submit your manuscript here: http://www.dovepress.com/patient-preference-and-adherence-journal 